\title{
Nucleus Striae Terminalis Lesions Affect Agonistic Behavior of Pigeons
}

\author{
J. MARTIN RAMIREZ AND JUAN D. DELIUS ${ }^{1}$ \\ Psychologisches Institut, Ruhr-Universität, Bochum \\ West Germany
}

\begin{abstract}
MARTIN RAMIREZ, J. AND J. D. DELIUS. Nucleus striae terminalis lesions affect agonistic behavior in pigeons. PHYSIOL. BEHAV. 22(5) 871-875, 1979.-Bilateral lesions of the nucleus striae terminalis suppressed the aggressive behavior shown by selected pigeons in response to a stick or the experimenter's hand being introduced into their home cage. Sham-operated animals did not show such an effect. Larger, accurately placed lesions led to an immediate suppression, smaller, less precisely placed lesions to a delayed effect. The suppression of aggression was accompanied by an increase in avoidance responses. The results are discussed in relation to suspected endocrinal activity of the nucleus striae terminalis and to the function this structure has in mammals.
\end{abstract}

Aggression Fear Lesions Nucleus striae terminalis Pigeon

THE mammalian amygdala and its avian homologue, the archistriatum, have convincingly been shown to play an important role in the control of agonistic behavior, even if the precise nature of this role still needs to be further elucidated (for reviews see $[9,19]$ ). Situated as it is at a high level of the neuraxis the amygdala/archistriatum must exert its influence through its efferent pathways, the most important being the stria terminalis. For mammals there is evidence that the nuclear masses on which this pathway terminates are similarly involved in steering aggressive and fear responses even though there is still much uncertainty about how the stria terminalis influences their functioning. For birds the information available on this issue is still fragmentary but the pattern may grossly conform with that found in mammals.

The present study focuses on the septal termination of the stria terminalis in the pigeon. In a previous investigation one of us [2] had found that electrical stimulation of the nucleus striae terminalis and its neighbourhood in awake, unrestrained gulls (Larus argentatus and fuscus) yielded threat behavior, sometimes accompanied by an increased tendency to respond aggressively to external stimuli for some fifteen minutes after the stimulation ended [7]. Histological peculiarities of the area in question, including an associated ependymal differentiation, suggested that the nucleus striae terminalis may be secreting into the lateral ventricle. Neurosecretory granules were later preliminarily verified in resorcin-fuchsin stained pigeon material. This led to the speculation that the release of a presumptive liquormone might have been responsible for the mood aftereffect [3]. We now simply began by examining the effect that lesions of the nucleus striae terminalis might have on the agonistic behavior of pigeons.

\section{METHOD}

Adult homing pigeons of unknown sex were bought from local dealers. All had previous free-ranging experience. In the laboratory they were kept in standard $50 \times 40 \times 30 \mathrm{~cm}$ cages. Blinds between the cages reduced the visual contact among the animals. They were exposed to a $12 \mathrm{hr}$ artificial light-12 hr dark cycle and to a nearly constant temperature of $18^{\circ} \mathrm{C}$ and had ad lib food and water. Before experiments began the animals had been kept in the laboratory for about one month.

In order to elicit agonistic responses from them the animals were exposed to two test stimuli. First the experimenter introduced a wooden stick into the cage, moving it with stabbing motions towards the animal for $30 \mathrm{sec}$ before withdrawing it. Fifteen seconds later he introduced his clenched fist into the cage and approached and followed the animal for $30 \mathrm{sec}$. After each bout of stimulation the experimenter recorded standardized scores and made notes about the behavioral responses the animal had shown. A description of the behavior and the details of the scoring will be given below. As a rule the animals were exposed to one testing sequence daily at 11 a.m. excepting weekends. Preliminary testing served to select 22 pigeons that responded reliably with aggressive (threat and attack) behavior. To qualify, each had to show such behavior in not less than $75 \%$ of at least 15 test pairs (stick and hand) given in the course of four weeks. These animals were then systematically tested once daily except weekends for a further week before they were submitted to surgery. After a postoperative recovery pause of one week they were tested for a further 8 weeks with a pause during the sixth week.

\footnotetext{
${ }^{1}$ Address reprint requests to J. D. Delius, Psychologisches Institut, Ruhr-Universität, 4630 Bochum, West Germany. While conducting this research J.M.R. was supported by a grant from the Alexander von Humboldt Stiftung and J.D.D. was aided by the Deutsche Forschungsgemeinschaft through its Sonderforschungsbereich 114. We are grateful to Mr. H. Stankewitz, Ms. G. Habers, Mrs. M. Hünecke and Dr. J. Emmerton for assistance in various matters.
} 
For the operation each animal was anaesthetized with Equithesin (intramuscular, initial dose $0.25 \mathrm{ml} / 100 \mathrm{~g}$ bodyweight, additional doses $0.1 \mathrm{ml} / 100 \mathrm{~g}$ ) and its head was held in a Stellar-Johnson stereotaxic apparatus modified according to the Karten and Hodos pigeon brain atlas [8]. The scalp was retracted and the skull perforated with a dental drill. A brass olive was placed in the cloaca, to serve as indifferent electrode. We attempted to place bilateral lesions in 16 birds at coordinates $A$ 6.75-7.75, L $1.75-2.25$ and $V$ 7.75-8.75 using an insulated stainless steel needle with a $0.5 \mathrm{~mm}$ exposed tip and by passing a $100 \mathrm{kHz}$ current of approximately $30 \mathrm{~mA}$ for $10 \mathrm{sec}$. Six control birds were similarly treated except that no coagulation current was applied.

After termination of the observations the animals were sacrificed and their brains were perfused with saline and Formalin following cannulation of the carotids. After at least one week fixation in Formalin the brains were removed from the calvarium and sectioned in the stereotactic frontal plane with a freeze microtome. The $40 \mu$ sections containing lesioned tissue were stained with cresyl violet and examined under a microscope. The extent of the lesions was transferred on to brain section outlines taken from the Karten and Hodos atlas [8].

\section{Behavior}

In the special test situation we used, the agonistic behavior of the pigeon differed somewhat from the descriptions found in the literature $[5,15]$. It is thus necessary to briefly review the behavior shown, leaning as far as possible on the accounts available.

The usually initial and least intense response to the testing stimuli is attention. Mostly with a latency of some seconds the subject takes up an erect posture, extending its neck, and begins to make scanning movements with the head, with the eyes wide open. It appears to watch the environment and particularly the experimental stimulus ready to respond with a more active behavior. If the neck remains stretched and the plumage sleeked during this attentive posture then it is likely that the subsequent behavior will be fearful; if the neck is shortened and the plumage fluffed, then the probable sequel is aggression. Freezing is a fear response that will often develop from the attentive response, the animal simply becoming immobile and remaining so for a period varying between several seconds and minutes before slowly beginning to make small amplitude movements or before suddenly exploding into extreme flight behavior. From attention or such unfreezing, pigeons often progress to leaning away, that is bending the body and head away from the stimulus with a sidewards orientation without shifting and while observing the stimulus intently. In this position it may hold out the wing closer to the stimulus: wing fending. This may lead to withdrawing, that is walking away, mostly with a sideward attitude to the stimulus, and attempting frantically to leave the cage, pushing against the wire netting. Attempts to $f l y$ away may then be observed. This active flight behavior is sometimes, though not often, preceded by alarm calls, harsh notes transcribable as "hinnh". A variant style of avoidance and escape is crouching, when the pigeon presses the body against the floor, the wings drooping slightly and the neck retracted, and sneaking, where it crawls away maintaining this posture.

A distant stimulus often elicits bow-cooing that is walking, tracing a series of reversing semicircles with the plumage fluffed, the tail spread and the throat swollen, with in- terspersed brief bursts of speed and body and head bows while giving repeated calls "cooo-cooo". If the stimulus approaches, the animal will usually revert to threat. The animal fluffs the plumage, sometimes also spreads the tail and swells the throat maintaining a sidewards orientation to the stimulus. The wing opposite to the stimulus is raised above the back, that close to the stimulus is flicked or more rarely also raised. This may lead to attack where the animal approaches the stimulus, sometimes jumping forward and slapping with the wing close to the stimulus, exceptionally with both wings, raising them and bring them down sharply on it. It will also peck, mostly short of target, but sometimes gripping the stimulus, vigorously shaking or twisting it with the eyes closed. A deep throated aggressive vocalization may accompany this behavior.

For the purpose of scoring, the response of a pigeon to a stick or hand test was classed as either attack, threat, retreat or flying away, depending on the responses predominantly shown by the animal. We will also refer to aggressive and avoidance behavior; these categories simply refer to attack and threat, and retreat and flying away respectively taken together. In a series of 32 test pairs the rating agreement between the routine observer (J.M.R.) and a cursorily instructed second observer was $88 \%$. Most non-matching judgements concerned the classes retreat-flying away. Referring to the categories aggression and avoidance, however, the agreement was $97 \%$. Additionally we also noted which of the behavior elements described above were shown during each test component. These elements, however, were not assessed in the interobserver reliability check. The results of both the hand and the stick test are reported because the spectrum of responses seen in the two tests differed somewhat as discussed in some detail elsewhere [9].

\section{RESULTS}

Of the 16 lesioned animals four, as later histology showed, had lesions that were misplaced. Another subject became ill and had to be discarded. The data presented refer to the remaining 11 pigeons. For evaluation the occurrence of the four test scores (or behavior elements) were expressed as percentages of the total number of tests administered in a week. Depending on the requirements, these percentages were averaged across animals and/or weeks as detailed below.

Six of the lesioned animals showed a marked reduction of aggressive behavior on being tested for the first time in the second week after the surgery. With a slight further enhancement the effect persisted throughout the observation period of eight weeks (Fig. 1). The change in behavior was nearly identical in all six individuals. Without exception they virtually ceased to show any attack or threat behavior to the test stimuli and instead responded predominantly with retreat behavior.

The five remaining lesioned animals showed a delayed effect. Only from the third post-operative week onwards was there a slight, but then steadily increasing, tendency for the animals to respond with retreat to the stick being introduced into their cage. The attack responses shown towards the experimenter's hand only began to revert from the fifth post-operative week onwards. Although this type of response predominanted during tests in the seventh and eighth post-operative weeks, the subjects even then continued to show some attack behavior during the hand tests (Fig. 1). In this group there was also some inter-individual variation 
amongst subjects when one compared their pre-operative behavior with the stabilized post-operative behavior shown in the seventh and eighth weeks of testing. One animal, for example, was striking in having switched from responding predominantly with attack to reacting mainly with flight rather than retreat.

The mean post-operative performance for each experimental individual was accordingly computed on a different basis for the two groups. For members of the group showing an immediate effect it was based on all post-operative tests. For the group showing a delayed effect it was based only on the performance of the two last weeks of testing. Statistical comparison of these post-operative measures of performance with the pre-operative measures indicated that both the decrease in aggression and the increase in avoidance was significant $(t$-tests, $d f=10$, aggression and avoidance, stick and hand tests, all $p<0.001$ ).

Analogously computed data relating to the frequency of

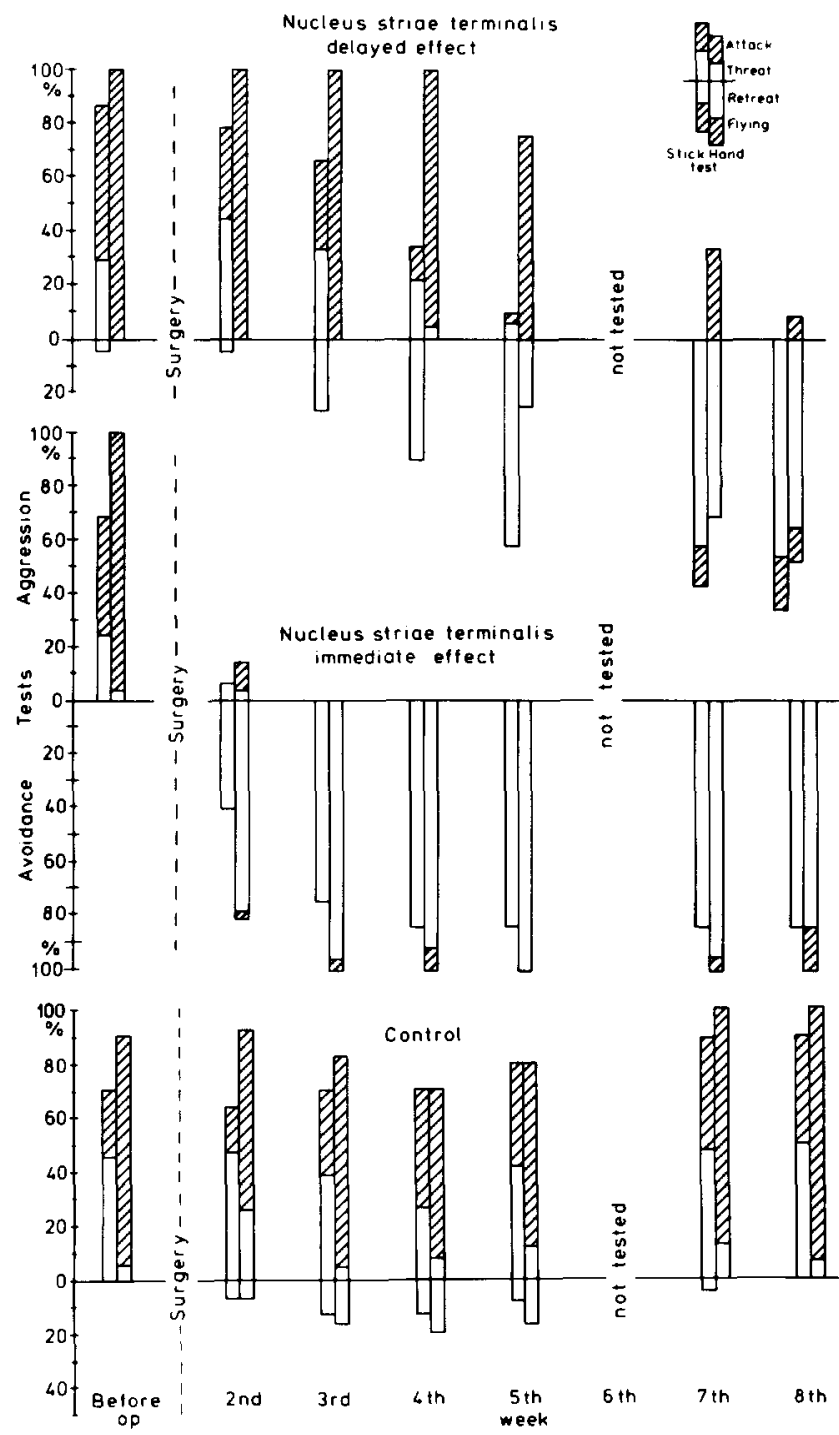

FIG. 1. Mean time course of agonistic behavior changes shown by pigeons after bilateral lesions of the nucleus striae terminalis. Six animals showed an immediate effect, five a delayed one. Data from six sham-operated controls is shown for comparison. occurrence of discrete agonistic behavior patterns were assembled and are shown in a summary way in Fig. 2. It indicates that virtually all the response patterns were affected in a way that agrees with the analysis based on the more gross test scores. Patterns of aggressive connotations were reduced, sometimes even eliminated, post-operatively whereas patterns implying avoidance, with the notable exception of freezing behaviour, were increased or even appeared de novo. Since these behaviors were largely the criteria by which the test scores were assigned, these data can not be viewed, however, as independently confirming the earlier analysis based on those scores.

The histological examination later revealed that the lesions in all 11 experimental animals were located on or in the immediate neighbourhood of the nucleus striae terminalis. In fact, of 4 additional pigeons in which the lesions had been misplaced, 3 had shown no behavioral effects while the remaining one, that had, turned out to have a very large medioventral hypothalamic lesion. Comparison of the group of animals that had shown an immediate behavioral effect with those which had yielded a delayed one indicated that there might be a correlation with the details of the extent and location of the lesions. Those belonging to the immediate group tended to have lesions mostly bilaterally centred on the relevant nuclei. They were also generally larger, particularly in anterior-posterior extent, a point that might be important in view of the increased extension in that axis that Zeier and Karten [22] allot to the nucleus striae terminalis in comparison with their earlier paper [21]. Pigeons belonging to the delayed group appeared to have suffered smaller coagulations sometimes only affecting one nucleus (Fig. 3).

Hardly any changes in behavior were seen in the shamoperated controls, only one animal showing a partial and transitory switch from aggressive to avoidance behavior during the post-operative period. Overall, however, there was no significant difference between the responses shown before and after surgery (Fig. $1 ; t$-tests, $d f=6$, aggression and avoidance, both stick and hand tests, $p>0.05$ ).

$$
\text { Nucleus striae terminalis }
$$

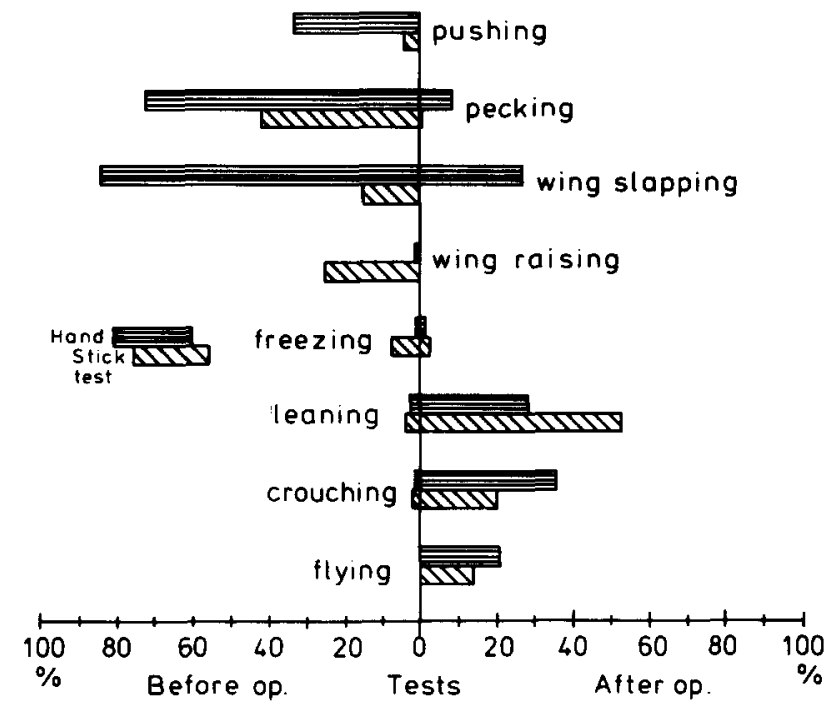

FIG. 2. Comparison of pre- and post-operative agonistic behavior of nucleus striae terminalis lesioned pigeons $(\mathrm{N}=11)$. 


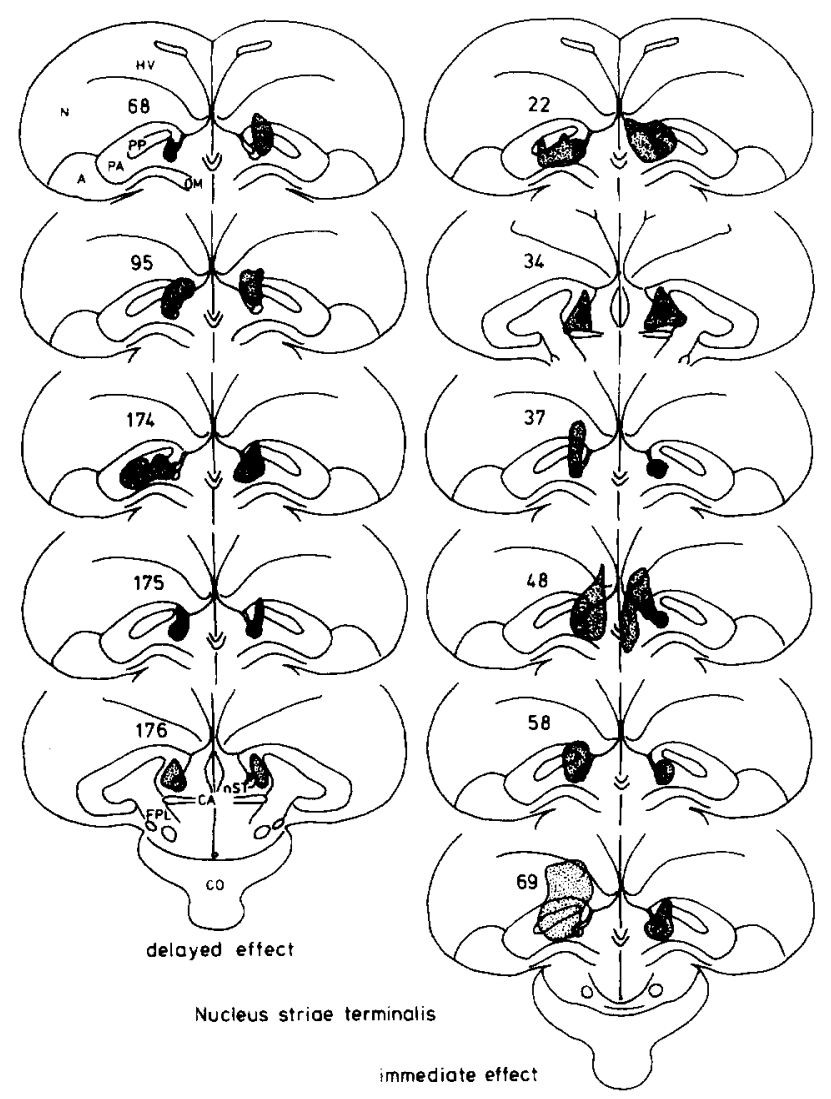

FIG. 3. Location of lesions in pigeons showing an immediate (right) and delayed (left) effect on agonistic behavior. Numbers identify subjects.

Relating to the food consumption, all groups showed a mild post-operative depression. This decrement was maintained somewhat longer in the lesioned animals but this difference was not statistically significant. The body weight rose slightly in all groups but most markedly in the lesioned group in spite of the diminished food intake. Again, however, the difference was not significant.

The results concerning the agonistic behavior are summarized in Fig. 4 which displays the average pre- and postoperative performance of the various groups. For the nucleus striae terminalis lesioned animals showing a delayed effect the average is based, as before, on the last two weeks of testing. Taking together the two lesioned groups and comparing their post-operative behavior with that of the shamoperated controls indicated that both the decrease in aggression and the increase in avoidance was significant $(t$-tests, $d f=15$; stick tests: aggression $p<0.005$, avoidance $p<0.001$, hand tests: aggression and avoidance both $p<0.001)$. The pre-operative behavior, as one might expect, did not differ significantly between the groups. Fig. 4 also displays the summary results of two further groups from an earlier study [9] involving lesions of the archistriatum and the overlying neostriatum caudale. As can be seen, the archistriatally lesioned pigeons yielded behavior changes similar to those seen in the nucleus striae terminalis lesioned ones whereas the neostriatals did not show any effect, being statistically indistinguishable from sham-operated animals.

\section{DISCUSSION}

Zeier and Karten [21], based on anatomical anterograde degeneration studies following archistrial lesions of the pigeon, described a nucleus situated at the level of the anterior commissure. It receives afferents from the posterior archistriatum via the occipito-mesencephalic tract and is homologous to the bed nucleus of the stria terminalis of mammals (compare, for example, [13]).

In line with findings in mammals, where electrical stimulation of the nucleus striae terminalis has been shown to evoke agonistic responses [6,19], one of us [2] found that stimulation of the same nucleus and its neighbourhood (as well as of the nucleus arcuatus hypothalami, known in birds as nucleus infundibuli) in herring and lesser black-backed gulls elicited threat behavior sequences. A review indicated that equivalent results had been obtained in other avian species, including the pigeon. A more recent compilation by Vowles and Beazley [18] confirms this by referring to a medial palaeostriatal strip just lateral to the telencephalic ventricles as particularly supporting electrically stimulated agonistic behavior in birds. The strip in question coincides closely with the extension of the nucleus striae terminalis mapped by Zeier and Karten [22].

As measured by the tests used in this study, lesions of the area in question suppress exogenously elicited aggression and facilitate avoidance behavior. This supports the contention that the avian bed nucleus of the stria terminalis is closely involved in the control of agonistic behavior. The relation that the somewhat artificial stimulation bears to the situations normally eliciting agonistic responses in pigeons has been discussed elsewhere [9]. Even though electrical stimulation of the bed nucleus elicits aggressive behavior in mammals, lesions of the same area do not appear to yield a clearcut suppression of aggression [19]. The issue is complicated, however, by the fact that lesions of the closely neighbouring medial septum often yield transitory increases in aggressiveness $([12,16]$; but see [1] and, regarding pigeons, [10]). Attention has now turned to the role that the nucleus might

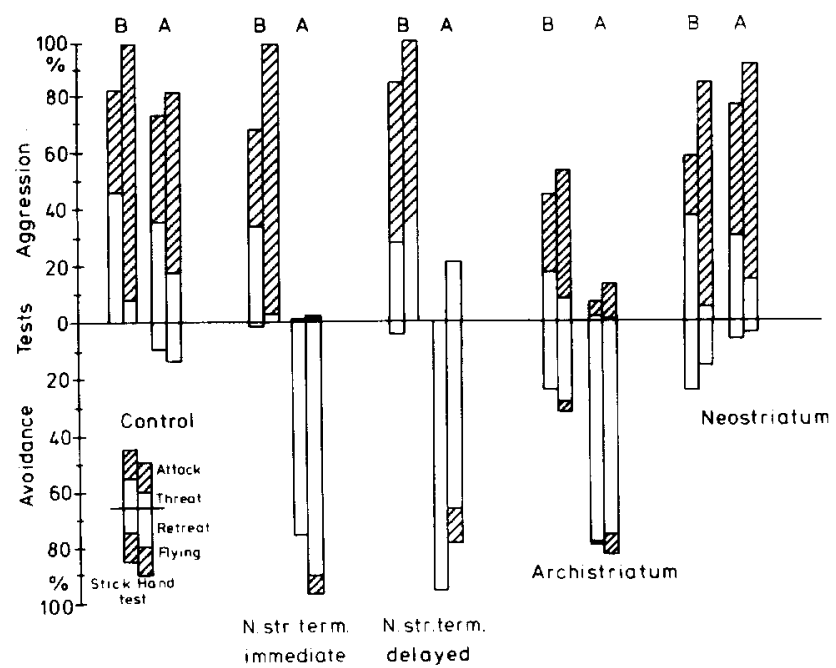

FIG. 4. Mean agonistic behavior shown by pigeons $(N=5$ or 6$)$ in each group during tests before (B) and after (A) variously located brain lesions and control sham operations. The post-operative behavior of $n$. striae terminalis, delayed-effect group is based on only the two last weeks of testing. 
have in the control of mammalian sexual behavior $[4,17]$.

The notion that the nucleus striae has an influence on aggressive behavior through ventricular neurosecretory activity $[2,3]$ remains open. The present results can not decide the issue. At least in some mammals, however, the neurosecretory function of the nucleus striae terminalis has in the meantime been confirmed. Neurons there have been shown to contain gonadotropin releasing hormone $[14,23]$. That this hypophysiotropic factor is also released into the mammalian cerebrospinal fluids seems likely since it is found there at reasonable titres [10]. Neurons sensitive to the hormone seem to be of widespread occurrence in these vertebrates [11]. Equivalent data for birds is not available and similarly it has not yet been shown that gonadotropin releasing hormone specifically influences aggressive behavior. It thus remains to be seen whether the releasing factor will fit the role of an aggression controlling liquormone that we suggest.

We must in this context consider whether the suppression of aggression, particularly the delayed suppression, we observed, might not have been caused by an endocrinal dysfunction. A secondary gonadotropin titre reduction, or even a tertiary gonadal hormone concentration decrease, could have been involved. Unfortunately we failed to ascertain the gonadal state of our experimental subjects. Nonetheless such a lengthy causal chain seems unlikely in the case of the immediate effect observed in one group of lesioned animals. However that might be, sufficient circumstancial evidence has now accrued to make it worthwhile to pursue the idea that a neurosecretion of the nucleus striae terminalis may somehow be involved in the regulation of aggressive behavior in birds.

\section{REFERENCES}

1. Blanchard, D. C., R. J. Blanchard, L. K. Takahashi and T. Takahashi. Septal lesions and aggressive behavior. Behav. Biol. 21: 157-161, 1977.

2. Delius, J. D. Agonistic behaviour of juvenile gulls, a neuroethological study. Anim. Behav. 21: 236-246, 1973.

3. Delius, J. D. Neuroetología de la agresión en aves. In: Progresos en Biología, VI Congreso Argentino de Biología. edited by F. D. Barbieri and A. H. Legñame. Tucumán: Miguel Lillo, 205-213, 1975.

4. Emery, D. E. and B. D. Sachs. Copulatory behaviour in male rats with lesions in the bed nucleus of the stria terminalis. Physiol. Behav. 17: 803-806, 1976.

5. Fabricius, E. and A. M. Jansson. Laboratory observations on the reproductive behaviour of the pigeon (Columba livia) during the preincubation phase of the breeding cycle. Anim. Behav. 11: $534-547,1963$.

6. Fernandez de Molina, A. and R. W. Hunsperger. Central representation of affective reactions in forebrain and brain stem: electrical stimulation of amygdala, stria terminalis and adjacent structures. J. Physiol. 145: 251-265, 1959.

7. Harwood, D. and D. M. Vowles. Defensive behaviour and the aftereffects of brain stimulation in the ring dove. Neuropsychologia 5: 345-366, 1967.

8. Karten, H. J. and W. A. Hodos. A Stereotaxic Atlas of the Brain of the Pigeon. Baltimore: Johns Hopkins Press, 1967.

9. Martin Ramirez, J. and J. D. Delius. Aggressive behaviour of pigeons: suppression by archistriatal lesions. Aggress. Behav., (in press).

10. Morris, M., B. Tandy, D. K. Sundberg and K. M. Knigge. Modifications of brain and CSF LH-RH following deafferentation. Neuroendocrinology 18: 131-135, 1975.

11. Moss, R. L. Role of hypophysiotropic neurohormones in mediating neural and behavioural events. Fedn Proc. 36: 1978$1983,1977$.

12. Nolley, D. A. Agonistic behaviour after stria terminalis lesion in the rat. Psychon. Sci. 28: 182-184, 1972.
13. Olmos, J. S. de and W. R. Ingram. The projection field of the stria terminalis in the rat brain, an experimental study.J. comp. Neurol. 146: 303-333, 1972.

14. Silverman, A. J. Distribution of luteinizing hormone-releasing hormone LHRH in the guinea pig brain. Endocrinology 99: 30-41, 1976.

15. Spiteri, N. Social, especially agonistic behaviour in the pigeon. M. Sc. Thesis, University of Durham, England, 1975.

16. Turner, B. H. Neural structures involved in the rage syndrome of the rat. J. comp. physiol. Psychol. 71: 103-113, 1970.

17. Velasco, M. E. and S. Taleisnik. Release of gonadotropins induced by amygdaloid stimulation in the rat. Endocrinology 84: $132-139,1969$.

18. Vowles, D. M. and L. D. Beazley. The neural substrate of emotional behaviour in birds. In: Birds: Brain and Behaviour, edited by I. J. Goodman and M. W. Schein. New York: Academic Press, 1974.

19. Zbrozyna, A. W. The organization of the defense reaction elicited from amygdala and its connections. In: The Neurobiology of the Amygdala, edited by B. E. Eleftheriou. New York: Plenum Press, 1972.

20. Zeier, $\mathbf{H}$. Changes in operant behaviour of pigeons following bilateral forebrain lesions. J. comp. physiol. Psychol. 66: 198$203,1971$.

21. Zeier, H. and H. J. Karten. The archistriatum of the pigeon: organization of afferent and efferent connections. Brain Res. 31: 313-326, 1971.

22. Zeier, H. and H. J. Karten. Connections of the anterior commissure in the pigeon (Columba livia). J. comp. Neurol. 150: 201-216, 1973.

23. Zimmermann, E. A. Localization of hormone secreting pathway in the brain by immunohistochemistry and light microscopy: a review. Fedn Proc. 36: 1964-1967, 1977. 